\title{
Simulation of 3D Image Reconstruction in Rigid body Motion
}

\author{
Huihong Chen ${ }^{1}$, Shiming Li*2 \\ ${ }^{1}$ Chen Huihong ( School of information engineering, Guangzhou Panyu polytechnic University, Guangzhou China, 511483) \\ ${ }^{2 *}$ Liu Shiming(Corresponding author) (School of management,Guangzhou Panyu polytechnic University, GuangZhou China, \\ 511483) \\ First author: Chen Huihong (June 1982), female, Han nationality, Fujian Gutian people, associate professor, master, mainly \\ engaged in image recognition, software development and big data research. \\ Second author(Corresponding author): Liu Shiming (December 1980), male, Han nationality, Hubei Zaoyang people, senior \\ engineer, doctor, mainly engaged in safety early warning, risk analysis and evaluation, safety management.
}

\begin{abstract}
D image reconstruction under rigid body motion is affected by rigid body motion and visual displacement factors, which leads to low quality of 3D image reconstruction and more noise, in order to improve the quality of 3D image reconstruction of rigid body motion. A 3D image reconstruction technique is proposed based on corner detection and edge contour feature extraction in this paper. Region scanning and point scanning are combined to scan rigid body moving object image. The wavelet denoising method is used to reduce the noise of the $3 \mathrm{D}$ image. The edge contour feature of the image is extracted. The sparse edge pixel fusion method is used to decompose the feature of the 3D image under the rigid body motion. The irregular triangulation method is used to extract and reconstruct the information features of the rigid body $3 \mathrm{D}$ images. The reconstructed feature points are accurately calibrated with the corner detection method to realize the effective reconstruction of the $3 \mathrm{D}$ images. The simulation results show that the method has good quality, high SNR of output image and high registration rate of feature points of image reconstruction, and proposed method has good performance of 3D image reconstruction.
\end{abstract}

\section{Introduction}

$3 \mathrm{D}$ image reconstruction is the basis of $3 \mathrm{D}$ visual reconstruction and virtual reality simulation in rigid body motion state. Through 3D image reconstruction in rigid body motion state, it provides a model foundation for building 3D visual simulation model, and the 3D image reconstruction of rigid body motion is carried out under computer vision, and virtual reality scene simulation platform is set up. A combination of computer technology, graphics and image technology, optical technology, control technology and other high technology to realize the reconstruction and Simulation of three-dimensional image information. In the design of 3D image reconstruction, three-dimensional modeling and related image processing algorithms are used to realize the simulation and visual reconstruction of the virtual reality scene. Three dimensional image reconstruction technology under dynamic state has very good application value in image processing, visual simulation and $3 \mathrm{D}$ modeling ${ }^{[1]}$.
3D image reconstruction is an important image processing technology in the field of computer vision. In the process of $3 \mathrm{D}$ image reconstruction under rigid body motion, because of the irregular shape of image itself and strong noise interference, it is difficult to reconstruct 3D images in traditional methods. Traditionally, 3D reconstruction of 3D images under rigid body motion can be divided into two categories: parametric statistical feature recombination model and geometric regular contour reconstruction model, $\operatorname{etc}^{[2]}$. The regional active contour model of 3D image in rigid body motion state is constructed by manually setting parameters. In sparse sampling point distribution technology, the homogeneous region of 3D image distribution under rigid body motion state is segmented ${ }^{[3,4]}$. The Mumford-Shah model is established for 3D image reconstruction under rigid body motion. In reference [5], a statistical shape model(SSM) is proposed for 3D image reconstruction under rigid body motion, and the statistical information feature reconstruction method is used to reconstruct 3D image. The image restoration and

*Corresponding author: 18520576074,31341517@qq.com/chenjin_2002@163.com 18520089909, hero640@163.com 
clarity processing of optical imaging under rigid body motion state is realized. The reconstruction accuracy is higher, but the reconstruction cost of the image with high interference intensity is higher. In reference [6], a structural model of 3D image reconstruction system based on active polarization imaging under rigid body motion is proposed. The reconstruction template shape is constructed and the similarity feature of target shape is extracted. The 3D image reconstruction under rigid body motion is carried out with the method of deformation displacement vector compensation. The disadvantage of this method is that the statistical information expression ability of reconstructed image is poor and the image is greatly affected by noise.

In order to solve the above problems, we propose a 3D image reconstruction technique based on corner detection and edge contour feature extraction. Firstly, the region scanning and point scanning are used to scan the rigid body moving object image. The wavelet denoising method is used to reduce the noise of the $3 \mathrm{D}$ image. The edge contour feature of the image is extracted. Then the sparse edge pixel fusion method is used to decompose the feature of the 3D image in the state of rigid body motion. The irregular triangulation method is used to extract and reconstruct the information features of the rigid body $3 \mathrm{D}$ images. The reconstructed feature points are accurately calibrated with the corner detection method to realize the effective reconstruction of the 3D images. Finally, the performance tests are carried out through simulation experiments, which demonstrate the superior performance of the proposed method in improving the performance of $3 \mathrm{D}$ image reconstruction under rigid body motion.

\section{3D Image acquisition and preprocessing in rigid body motion}

\subsection{Image scanning of moving objects of rigid bodies}

In order to realize $3 \mathrm{D}$ image reconstruction under rigid body motion, we use the region scanning and point scanning technology to scan the image of rigid body moving object, and realize the global spatial scanning of reconstructed object. The spatial scanning methods such as Cell Projection and Splattingare are used to analyze the random and deterministic distribution of 3D images in rigid body motion ${ }^{[7]}$. The arbitrary pixel values from the beginning are selected as the initial values of the parameters of the global spatial scanning. The laser intensity attenuation coefficient is set and the image is replaced by column and column. Matrixes $\mathbf{Q}, \mathbf{Q}^{r}$ and $\mathbf{Q}^{r c}$ are used to represent the $M \times N$ dynamic image of rigid body, gray image and sub-template of image respectively ${ }^{[8]}$.

According to the edge contour calibration, subpixel matching method is used to compensate the recognition error caused by the dynamic difference of rigid body motion. The sparse linear equations of image template matching under rigid body motion are obtained as follows:

$$
g(x, y)=h(x, y) * f(x, y)+\eta(x, y)
$$

Where, $h(x, y)$ is the parallax function and the symbol * denotes convolution. According to the image pixel level parallax function, the beamspace with parallax is solved, and the subpixel parallax is obtained in the $3 \mathrm{D}$ image scanning direction under rigid body motion:

$$
g(x, y)=f(x, y)+\eta(x, y)
$$

Where, $\eta(x, y)$ is the gray value of the energy function about parallax. In the case of image reconstruction and contour with high intensity noise interference, the difference pixel characteristics contained in each sub-region information are analyzed by image filtering ${ }^{[9]}$. The data items and smoothing items of 3D image feature extraction under rigid body motion are calculated, and the edge pixel estimates are obtained as follows:

$$
\hat{f}(x, y)=\beta F(x, y)+(1-\beta) m_{l}+\delta_{l}^{2}
$$

Where, $F(x, y)$ is the strong texture set of $3 \mathrm{D}$ images in rigid body motion, $(x, y)$ is the weak texture set and $m_{l}$ is the local variance.

The gradient information of 3D image under rigid body motion is obtained by combining spatial scanning of Ray-Casting image. In 3D data field, the imaging region of $3 \mathrm{D}$ image scanning under rigid body motion is divided into two homogeneous regions (object and background). The image sequence is approximated in subspace in the direction of line of sight. Assuming that the color information $I(x, y)$ of $\Omega$ is divided into two non-overlapping regions $R_{1}$ and $R_{2}$ by ray casting curve $\mathrm{G}$, the matching point neighborhood of sampling points is calculated, and the mesh model of $3 \mathrm{D}$ image reconstruction under rigid body motion is obtained ${ }^{[10]}$. The matching function between the sampling points and the vertices of the grid model is obtained by scanning the Ray-Casting space as follows:

$$
\begin{aligned}
& E^{c v}\left(c_{1}, c_{2}\right)=\mu \cdot \text { Length }(C) \\
& +v \cdot \operatorname{Area}(\operatorname{inside}(C)) \\
& +\lambda_{1} \int_{\text {inside }(C)}\left|I-c_{1}\right|^{2} d x d y \\
& +\lambda_{2} \int_{\text {outside }(C)}\left|I-c_{2}\right|^{2} d x d y
\end{aligned}
$$

Where, $c_{1}$ and $c_{2}$ represent spatially distributed thread primitives of 3D images in rigid body motion state of target and background region respectively, Length $(C)$ denotes regional distributed contour length, $\operatorname{Area}(\operatorname{inside}(C))$ denotes pixel intensity, and $\lambda_{1}$ and $\lambda_{2}$ denote spatial sweep of each image. The weight coefficients of 
constraint vectors are constants greater than 0 . Through the spatial scanning of the reconstructed Ray-Casting image, the original image input is provided for the 3D reconstruction of the image ${ }^{[11]}$.

\subsection{D image denoising processing}

On the basis of using region scanning and point scanning to scan rigid body moving object image, the paper carries out three dimensional image denoising processing. In this paper, wavelet denoising method is used to do three dimensional image de-noising processing ${ }^{[12]}$. In the state of volume motion, 3D image is decomposed by wavelet decomposition. The closed curve of wavelet is $C$, and expressed as $C=\{(x, y) \in \Omega: \phi(x, y)=0\} \quad$ by a binary gradient function. In the active region of contour line, the holographic projection image of 3D image under rigid body motion is obtained, coefficient $v$ is usually 0 , where the value is 0 :

$$
\begin{aligned}
& E^{c v}\left(c_{1}, c_{2}\right)= \\
& \mu \int_{\Omega} \delta(\phi(x, y))|\nabla \phi(x, y)| d x d y \\
& \quad+\lambda_{1} \int_{\Omega}\left|I-c_{1}\right|^{2} H(\phi(x, y)) d x d y \\
& \quad+\lambda_{2} \int_{\Omega}\left|I-c_{2}\right|^{2}(1-H(\phi(x, y))) d x d y
\end{aligned}
$$

Where, $H(z)$ and $\delta(z)$ are the regular terms of the pixel sparsity in the region of the three-dimensional image noise in the rigid body motion state respectively. By the regularized Dirac decomposition, the noise reduction filtering of the three-dimensional image under the rigid body motion state is calculated ${ }^{[13]}$, and the energy function of the pixel feature distribution of the image is obtained as follows:

$$
\begin{aligned}
& E^{L B F}\left(\phi, f_{1}, f_{2}\right)= \\
& \quad \mu \int \frac{1}{2}(|\nabla \phi|-1)^{2} d x+v \cdot \text { Length }(C) \\
& \quad+\lambda_{1} \int\left[\int K_{\sigma}(x-y)\left|I-f_{1}(x)\right|^{2} H(\phi) d y\right] d x \\
& \quad+\lambda_{2} \int\left[\int K_{\sigma}(x-y)\left|I-f_{2}(x)\right|^{2}(1-H(\phi) d y)\right] d x
\end{aligned}
$$

Where, $\lambda_{1}, \lambda_{2}, \nu, \mu$ are a non-negative constant, $K_{\sigma}$ is the standard deviation of Heaviside function, $\sigma$ is the weight coefficient, and the size of the weight coefficient $\mathrm{R}$ is chosen to adjust the size of the reconstructed edge contour of the $3 \mathrm{D}$ image in rigid body motion. A set constraint item is used to ensure the stability of the level set function, and the local gradient energy distribution function is established by using the edge contour information of the image, which can effectively distinguish the template of the imaging object and the background area of the 3D image, and set up the local image location. The partial binary fitting term is used to fit the local energy. The gray values of $3 \mathrm{D}$ image reconstruction are represented by $f_{1}(x)$ and $f_{2}(x)$. In the horizontal concentration of pixel distribution in background area, $f_{1}(x)$ and $f_{2}(x)$ change with spatial domain.

Wavelet subspace fusion method is used to reduce noise. The output of the feature components of the pixel fusion region of the image is as follows:

$$
I(x)=J(x) t(x)+A(1-t(x))
$$

Where, $A$ is the scale information of 3D image reconstruction in a single frame of rigid body motion, $t(x)$ is the distribution density of noise, and $J(x) t(x)$ is the distribution distance between edge contour point and information center point ${ }^{[14]}$.

\section{$33 \mathrm{D}$ image reconstruction}

\subsection{D image feature decomposition}

In this paper, a 3D image reconstruction technique is proposed based on corner detection and edge contour feature extraction. The sparse edge pixel fusion method is used to realize $3 \mathrm{D}$ image feature decomposition and knot in rigid body motion. With the gradient information of the local image, the 3D coordinates of the image distributed data volume in the motion state of rigid body are obtained by wavelet analysis. The contour length of the $3 \mathrm{D}$ reconstruction of the image is as follows:

$$
E=\theta E^{L B F}+(1-\theta) E^{L G F}+v L(\phi)+\mu P(\phi)
$$

Where, $\theta$ is the gray weight coefficient of each pixel neighborhood, $L(\phi)$ is the constraint item of edge contour length, the target region and background area are smoothed, and the box model is defined as follows:

$$
L(\phi)=\int_{\Omega} \delta(\phi)|\nabla \phi| d x
$$

Where, $P(\phi)$ is a sparse canonical term, the local Gao Si probability distribution of edge pixels is obtained by bounding box method as follows:

$P(\phi)=\int \frac{1}{2}(|\nabla \phi|-1)^{2} d x$

Where, $E^{L B F}$ is the local gray level information, $E^{L G F}$ is the local gradient energy term, thus the 3D coordinate of the ray entering the data body is defined as follows:

$$
\begin{aligned}
& E^{L B F}\left(\phi, f_{1}, f_{2}\right)= \\
& \lambda_{1} \int\left[\int K_{\sigma}(x-y)\left|I-f_{1}(x)\right|^{2} H(\phi) d y\right] d x \\
& +\lambda_{2} \int\left[\int K_{\sigma}(x-y)\left|I-f_{2}(x)\right|^{2}(1-H(\phi)) d y\right] d x
\end{aligned}
$$




$$
\begin{aligned}
& E^{L G F}\left(\phi, f_{1}^{G}, f_{2}^{G}\right)= \\
& \lambda_{1} \int\left[\int K_{\sigma}(x-y)\left|I^{G}-f_{1}^{G}(x)\right|^{2} H(\phi) d y\right] d x+ \\
& \lambda_{2} \int\left[\int K_{\sigma}(x-y)\left|I^{G}-f_{2}^{G}(x)\right|^{2}(1-H(\phi)) d y\right] d x
\end{aligned}
$$

In the formula, $I^{G}$ denotes the gradient mode of the reconstructed surface, and $f_{1}^{G}$ and $f_{2}^{G}$ represent the gradient mode of the curved part and the linear part of the reconstructed mesh surface. In this way, the $3 \mathrm{D}$ data field of image reconstruction is constructed, and the sparse edge pixel fusion method is used to realize the $3 \mathrm{D}$ image feature decomposition under rigid body motion, and the characteristic decomposition equation is obtained as follows:

$$
\begin{aligned}
\frac{\partial \phi}{\partial t}= & -\delta(\phi)\left[\theta\left(\lambda_{1} e_{1}^{L B F}-\lambda_{2} e_{2}^{L B F}\right)\right. \\
& \left.+(1-\theta)\left(\lambda_{1} e_{1}^{L G F}-\lambda_{2} e_{2}^{L G F}\right)\right] \\
& +v \delta(\phi) \operatorname{div}\left(\frac{\nabla \phi}{|\nabla \phi|}\right) \\
& +\mu\left(\nabla^{2} \phi-\operatorname{div}\left(\frac{\nabla \phi}{|\nabla \phi|}\right)\right)
\end{aligned}
$$

Where, $e_{1}^{L B F}, e_{2}^{L B F}, e_{1}^{L G F}, e_{2}^{L G F}$ are calculated as:

$\left\{\begin{array}{l}e_{1}^{L B F}=\int_{\Omega} K_{\sigma}(y-x)\left|I(x)-f_{1}(y)\right|^{2} d y \\ e_{2}^{L B F}=\int_{\Omega} K_{\sigma}(y-x)\left|I(x)-f_{2}(y)\right|^{2} d y\end{array}\right.$

$\left\{\begin{array}{l}e_{1}^{L G F}=\int_{\Omega} K_{\sigma}(y-x)\left|I^{G}(x)-f_{1}^{G}(y)\right|^{2} d y \\ e_{2}^{L G F}=\int_{\Omega} K_{\sigma}(y-x)\left|I^{G}(x)-f_{2}^{G}(y)\right|^{2} d y\end{array}\right.$

Where, $H(\phi)$ is a Heaviside function, representing the spatial mapping of $3 \mathrm{D}$ image distribution under rigid body motion, and $\delta(z)=\frac{d}{d z} H(z)$ is a Dirac function, representing the dynamic feature points of rigid body motion.

\subsection{D image reconstruction output}

The irregular triangulation method is used to extract and reconstruct the information feature of the rigid body 3D image. We set $\left\{w_{1}, w_{2}, \ldots, w_{d_{i}}\right\}$ denote the vector weight of the $3 \mathrm{D}$ image reconstruction extraction under the rigid body motion state, and assume the 3D image weight under the rigid body motion state. The length of the location information association distribution is $L=x_{\max }-x_{\min }$, the width is $W=y_{\text {max }}-y_{\text {min }}$, and the height is $H=z_{\text {max }}-z_{\text {min }}$. By using the three-dimensional space reconstruction method, the retrieval topology of 3D image reconstruction under rigid body motion is represented by the following four vector quantization functions $x_{1}, x_{2}, x_{3}$ and $x_{4}$ :

$$
\left\{\begin{array}{l}
x_{1}=p_{1}-m \\
x_{2}=p_{2}-m \\
x_{3}=p_{3}-m \\
x_{4}=m
\end{array}\right.
$$

Where, $m$ is the edge segmentation scale of $3 \mathrm{D}$ image reconstruction under rigid body motion in adjacent domain, and the affine moment of 3D image reconstruction optical image region segmentation single frame information $I(x, y)$ in the whole reconstructed geometric space under rigid body motion condition. $\bar{\eta}$ and pixel invariant moments $\widehat{R}_{x}$, whose estimated values are:

$$
\widehat{\eta}=\arg \min _{\eta} L(\eta), \widehat{R}_{x}=\frac{1}{K} \sum_{k=1}^{K} x_{k} x_{k}{ }^{H}
$$

Where, $L(\eta)$ represents the binary expression function of the rigid body moving image, and the edge of the image is segmented by binary processing, and the edge trajectory of the image is obtained. The $x_{k}$ denotes the input pixel level, and the $K$ represents the pixel level:

$$
\tilde{t}(x)=1-\min _{c}\left(\min _{y \in \Omega(x)}\left(\frac{I^{c}(y)}{A^{c}}\right)\right)
$$

Where, $I^{c}(y)$ is the initial pixel set, and $A^{c}$ represents the scale information of the image. According to the above processing, the irregular triangulation method is used to extract and reconstruct the information features of the rigid body $3 \mathrm{D}$ image. The moving object image reconstruction is realized $^{[15]}$, and the edge pixel set of the $3 \mathrm{D}$ image reconstruction under the rigid body motion is obtained as follows:

$$
\begin{aligned}
& I_{i f}(x, y)=I * G\left(x, y, \sigma_{i}\right) \\
& I_{i v}(x, y)=I * \operatorname{stdfilt}\left(x, y, w_{i}\right) \\
& S_{g i f}(x, y)=-\log \left(P_{i f}(x, y)\right)
\end{aligned}
$$

Where, $G\left(x, y, \sigma_{i}\right)$ denotes the high frequency component set of 3D image reconstruction in $4 \times 4$ subgrid region under rigid body motion. According to the result of image reconstruction, 3D image reconstruction in rigid body state is carried out. Based on the above processing, the reconstructed feature points are accurately calibrated with corner detection method, and the effective reconstruction of 3D images is realized. The implementation flow of the improved algorithm is shown in figure 1.

\section{Simulation test and result analysis}

In order to test the application performance of this method in 3D image reconstruction under the rigid body motion state, we carry out the simulation 
experiment. The Matlab 7 simulation software is designed, and the Laser 5 3D image scanner is used to scan the image of the rigid body motion state. The training sample size of the rigid body motion image is 1200 , and the frame of the moving image is made. The frequency of the scanning is $12 \mathrm{KHz}$, the time interval of the $3 \mathrm{D}$ image sampling is $t=4 \mathrm{~s}$, the dimension of the 3D image reconstruction region is divided into $m^{*} n$ bit $360 * 240$, the width of the pixel window $(16,32,64)$, the resolution of the $3 \mathrm{D}$ image reconstruction in the rigid body motion state is

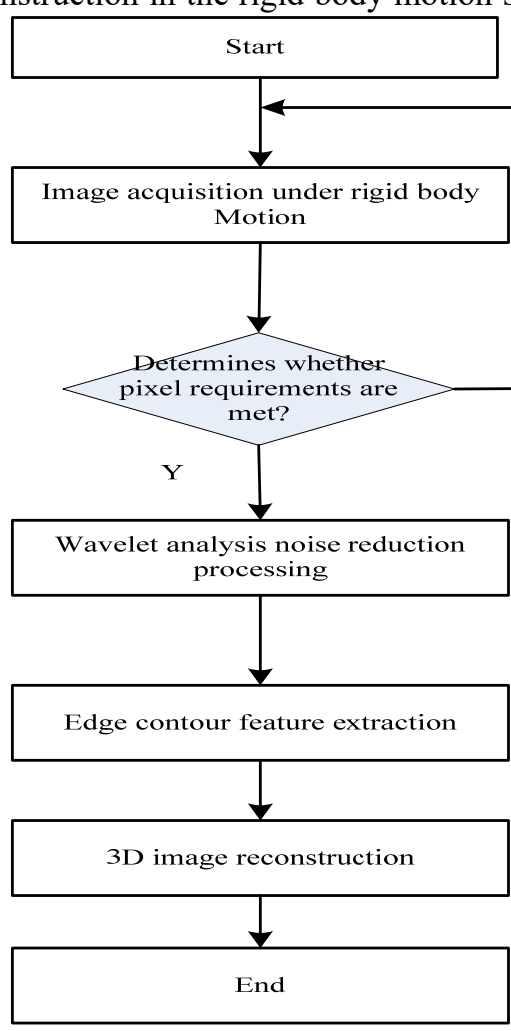

Figure 1. Process of 3D image reconstruction under rigid body motion

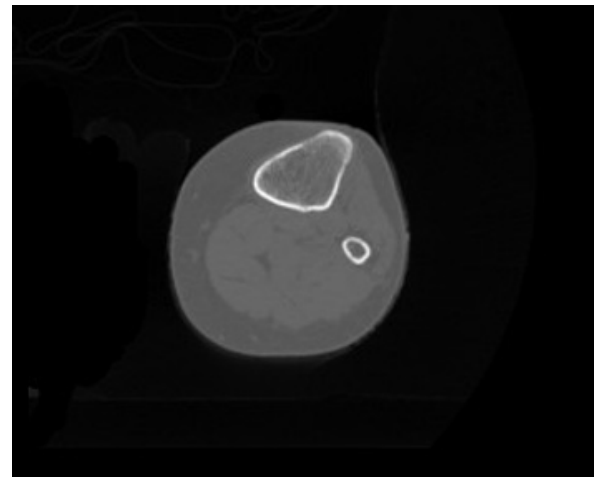

Figure 2. Three-dimensional image under rigid body motion

The 3D image in the rigid body motion state of figure 2 is taken as the research object, the $3 \mathrm{D}$ image reconstruction is carried out, and the wavelet denoising method is used to de-noise the image, and the edge contour feature is extracted from the de-noised image. The result of feature extraction is as shown in Figure 3.
$1200 * 1600$, the edge coefficient of the 3D image reconstruction is $\mu=0.021 * 232 * 200$, the sparsity $v=1$, the image reconstruction. The time interval $\Delta t=0.24$ and the correlation coefficient of the image distribution are $\mathcal{E}=0.125$. According to the simulation environment and parameters set above, the 3D image reconstruction simulation of the rigid body motion state is carried out. The original collection of rigid body motion images is shown as shown in Figure 2 .

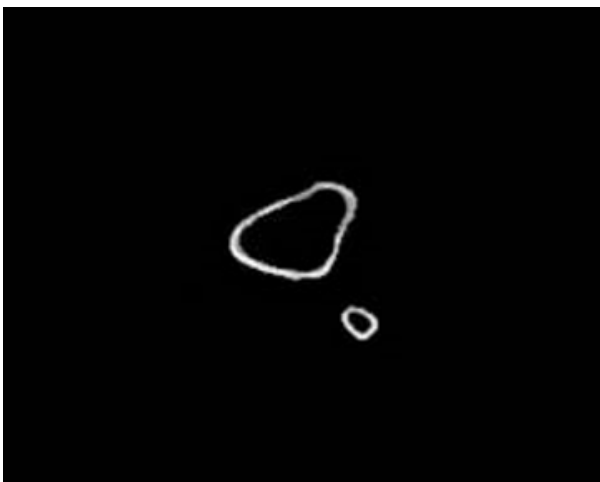

Figure 3. Image edge contour feature extraction The irregular triangulation method is used to extract and reconstruct the information feature of the rigid body $3 \mathrm{D}$ image, and the reconstructed output is shown in figure 4. 


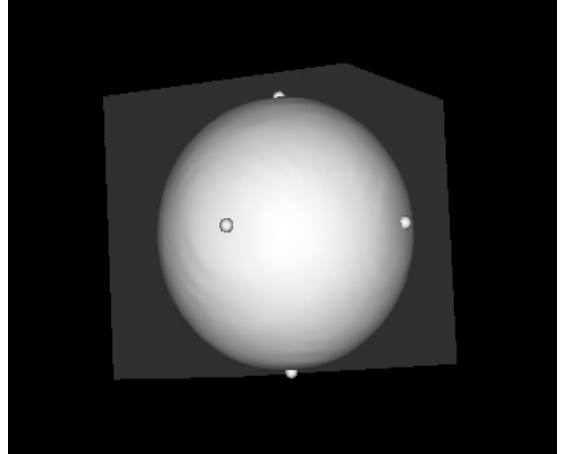

Figure 4. Result of 3D image reconstruction

The analysis of Figure 4 shows that the quality of $3 \mathrm{D}$ image reconstruction in rigid body motion is better and the registration rate of the feature points of the image reconstruction is high. In order to compare the quality of the reconstruction of the image, we use the signal to noise ratio of the image as the test index, and the contrast results are shown as shown in Figure 5 .

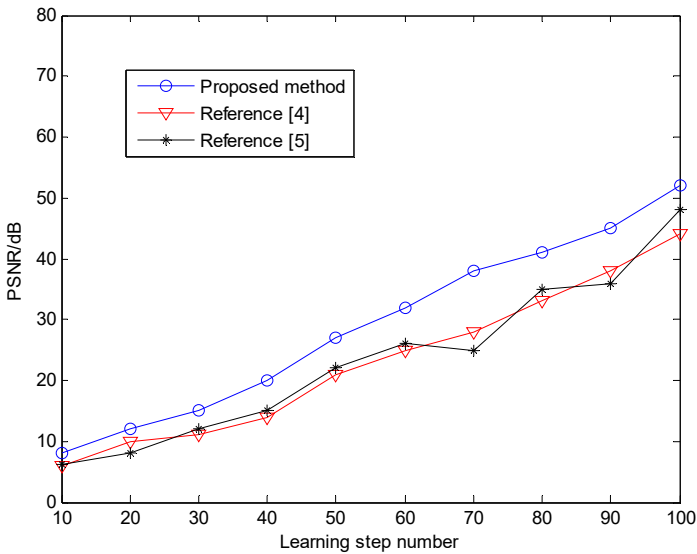

Figure 5. Comparison of output signal-to-noise ratio of image reconstruction

The simulation results show that the proposed method has better quality and higher PSNR of the output images under rigid body motion, which is $12.5 \%$ and $21.3 \%$ higher than that of the traditional methods. Therefore, we can see that the reconstruction effect of this method is better.

\section{Conclusions}

In this paper, we propose a 3D image reconstruction technology based on corner detection and edge contour feature extraction. The image of the moving object is scanned by regional scanning and point scanning, and then the wavelet denoising method is used to denoise the three-dimensional image, thus the edge contour features of the image are extracted. According to the feature of the contour, the feature of three-dimensional image under the rigid body motion is decomposed by the sparse edge pixel fusion method, and the information feature of the 3D image is extracted and reconstructed by the irregular triangulation. Then, the corner detection method is used to calibrate the reconstructed feature points, and finally the 3D image can be reconstructed effectively. The simulation results show that the method has good image quality, high output signal to noise ratio and high registration rate of image reconstruction feature points, and has good performance of 3D image reconstruction. This method has good application value in $3 \mathrm{D}$ reconstruction of rigid motion images.

\section{Project information :}

1. Guangzhou education system innovation academic team project, numbered: 1201610034, project name: pedestrian detection and tracking innovation team in complex visual scene

2. The ninth batch of Guangzhou City educational reform project, number: 2017F06, the name of the project: creating apprenticeship system in workshop practice

\section{References}

1. WANG Xin, ZHOU Yun, NING Chen, SHI Aiye. Image saliency detection via adaptive fusion of local and global sparse representation[J]. Journal of Computer Applications, 2018, 38(3): 866-872.

2. GOFERMAN S, ZELNIKMANOR L, TAL A. Context-aware saliency detection[J]. IEEE Transactions on Pattern Analysis and Machine Intelligence, 2012, 34(10):1915-1926.

3. CHENG M M, MITRA N J, HUANG X, et al. Global contrast based salient region detection[J]. IEEE Transactions on Pattern Analysis and Machine Intelligence, 2015, 37(3):569-582.

4. LIU N, HAN J. DHSNet:deep hierarchical saliency network for salient object detection[C]//Proceedings of the 2016 IEEE Conference on Computer Vision and Pattern Recognition. Washington, DC:IEEE Computer Society, 2016:678-686.

5. KIM W, KIM C. Spatiotemporal saliency detection using textural contrast and its applications[J]. IEEE Transactions on Circuits \& Systems for Video Technology, 2014, 24(4):646-659.

6. YAN Q, XU L, SHI J, et al. Hierarchical saliency detection[C]//CVPR'13:Proceedings of the 2013 IEEE Conference on Computer Vision and Pattern Recognition. Washington, DC:IEEE Computer Society, 2013:1155-1162.

7. WANG X, NING C, XU L. Spatiotemporal saliency model for small moving object detection in infrared videos[J]. Infrared Physics \& Technology, 2015, 69:111-117.

8. LI Xiao, GE Baozhen, LUO Qijun, LI Yunpeng, TIAN Qingguo. Acquisition of camera dynamic extrinsic parameters in free binocular stereo vision syste[J]. Journal of Computer Applications, 2017, 37(10): 2888-2894. 
9. ZHANG Quangui, CAI Feng, LI Zhiqiang. Human action recognition based on coupled multi-Hidden Markov model and depth image data. Journal of Computer Applications, 2018, 38(2): 454-457.

10. LI Songlin, JIA Yong, GUO Yong, ZHONG Xiaoling, CUI Guolong. Moving target tracking algorithm based on improved Camshift for through-wall-radar imaging $[\mathrm{J}]$. Journal of Computer Applications, 2018, 38(2): 528-532.

11. TAN Q Y, LEUNG $\mathrm{H}$, SONG $\mathrm{Y}$, et al. Multipath ghost suppression for through-the-wall-radar[J]. IEEE Transactions on Aerospace and Electronic Systems, 2014, 50(3):2284-2292.

12. HU Min, LI Chong, LU Rongrong, HUANG Hongcheng. Performance analysis of motor imagery training based on $3 \mathrm{D}$ visual guidance[J]. Journal of Computer Applications, 2018, 38(3): 836-841.

13. ONO T, KIMURA A, USHIBA J. Daily training with realistic visual feedback improves reproducibility of event-related desynchronisation following hand motor imagery[J]. Clinical Neurophysiology, 2013, 124(9):1779-1786.

14. REN Shuai, ZHANG Tao, XU Zhenchao, WANG Zhen, HE Yuan, LIU Yunong. Information hiding algorithm for 3D models based on feature point labeling and clustering[J]. Journal of Computer Applications, 2018, 38(4): 1017-1022.

15. HUA S G, ZHONG Q, LI S S. 3D shape deformation based on edge collapse mesh simplification[J]. Journal of Dalian University of Technology, 2011, 51(3):363-367. 